\title{
Renormalized Solutions for Nonlinear Degenerate Elliptic Problems with $L^{1}$ Data
}

\author{
Kaouther AMmAR and Hicham RedwANe
}

\author{
TU Berlin \\ Institut für Mathematik, MA 6-3 \\ Strasse des 17. Juni 136 \\ 10623 Berlin - Germany \\ ammar@math.tu-berlin.de
}

\author{
Faculté des Sciences Juridiques, \\ Economiques et Sociales \\ Université Hassan 1 \\ B.P. 784. Settat - Morocco \\ redwane_hicham@yahoo.fr
}

Received: August 27, 2007

Accepted: January 29, 2008

\section{ABSTRACT}

We are interested in a class of nonlinear degenerate diffusion problems with a diffusion function $a(x, u, \nabla u)$ which is not controlled with respect to $u$ and which is not uniformly coercive on the weighted Sobolev spaces $W_{0}^{1, p}(\Omega, w)$. Existence of a renormalized solution is proved in the $L^{1}$-setting.

Key words: Renormalized solutions, nonlinear degenerate elliptic equations, weighted Sobolev spaces.

2000 Mathematics Subject Classification: 47A15, 46A32, 47D20.

\section{Introduction}

In this paper we investigate the problem of existence of renormalized solutions for a class of nonlinear degenerate elliptic equations of the type

$$
\begin{aligned}
-\operatorname{div}(a(x, u, \nabla u)) & =f & & \text { in } \Omega, \\
u & =0 & & \text { on } \partial \Omega,
\end{aligned}
$$

where $\Omega$ is an open bounded subset of $\mathbb{R}^{N}, N \geq 1$, and the data $f$ is in $L^{1}(\Omega)$. The operator $-\operatorname{div}(a(x, u, \nabla u))$ is a Leray-Lions operator defined on the weighted 
Sobolev spaces $W_{0}^{1, p}(\Omega, w)$, but which is not controlled with respect to $u$ (see assumptions (5), (7), and (8) of section 2). For almost any $x$ in $\Omega$ and for any $\xi \in \mathbb{R}^{N}$, the function $a(x, s, \xi)$ is strongly degenerate when $|s|$ grows to $+\infty$ (see (6) and (7)). So, proving existence of a weak solution (i.e., in the distribution meaning) seems to be an arduous task. To overcome this difficulty we use in this paper the framework of renormalized solutions. This notion was introduced by P.-L. Lions and Di Perna [12] for the study of the Boltzmann equation (see also P.-L. Lions [15] for a few applications to fluid mechanics models). This notion was then adapted to the elliptic version of (1) and (2) in Boccardo, J.-L. Diaz, D. Giachetti, F. Murat [11], in P.-L. Lions and F. Murat [16], and F. Murat [16,17] (see also [8,9] for nonlinear parabolic problems). At the same time the equivalent notion of entropy solutions have been developed independently by Bénilan and al. [5] for the study of nonlinear elliptic problems.

In the case where $a(x, u, \nabla u)$ is replaced by $A(x, u) \nabla u$ (problems with diffusion matrix which are not uniformly coercive with respect to $u$ ) and $f \in L^{1}(\Omega)$, existence and a partial uniqueness result have been established on the Sobolev spaces $H_{0}^{1}(\Omega)$ in D. Blanchard and O. Guibé [6] (see also D. Blanchard, O. Guibé, and H. Redwane [7], K. Ammar [4]).

Note that in the non weighted case, the existence and regularity results for the nonlinear elliptic problem (1), (2) has been proved in A. Alvino, L. Boccardo, V. Ferone, L. Orsina, and G. Trombetti [2] under the condition $a(x, s, \xi) \xi \geq \frac{\alpha}{(1+|s|)^{\theta(p-1)}}|\xi|^{p}$ and under various assumptions on the function $f$ and on $\theta$, (see also the results of A. Alvino, V. Ferone, and G. Trombetti [3], L. Boccardo, A. Dall'Aglio, and L. Orsina [10]).

In our paper we propose a formulation which takes into account the possible values $+\infty$ or $-\infty$ for the solutions and the operator $-\operatorname{div}(a(x, u, D u))$ is a weighted Leray-Lions operator from the weighted Sobolev space on $W_{0}^{1, p}(\Omega, \omega)$ into $W^{-1, p^{\prime}}\left(\Omega, \omega^{*}\right)$.

The paper is organized as follows: In section 1, we precise some basic properties of weighted Sobolev spaces. In section 2, we specify the assumptions on $a(x, s, \xi), b(s)$ and $f$ needed in the present study and we give the definition of a renormalized solution of (1), (2). In section 3, we prove the main result of this paper (Theorem 3.1) which is the existence of a renormalized solution for any data $f$ in $L^{1}(\Omega)$.

\section{Preliminaries}

Throughout the paper, we assume that the following assumptions hold true: $\Omega$ is a bounded open subset on $\mathbb{R}^{N}, N \geq 1,1<p<\infty$, and $\omega(x)=\left\{\omega_{i}(x)\right\}_{\{0 \leq i \leq N\}}$ is a vector of weight functions. Further, we suppose that every component $\omega_{i}(\bar{x})$ is a measurable function which is strictly positive and satisfies

$$
\omega_{i} \in L_{\mathrm{loc}}^{1}(\Omega) \quad \text { and } \quad \omega_{i}^{-\frac{1}{p-1}} \in L_{\mathrm{loc}}^{1}(\Omega) .
$$


We define the weighted Lebesgue space $L^{p}\left(\Omega, \omega_{0}\right)$ with weight $\omega_{0}$, as the space of all real-valued measurable functions $u$ for which

$$
\|u\|_{p, \omega_{0}}=\left(\int_{\Omega}|u(x)|^{p} \omega_{0}(x) d x\right)^{\frac{1}{p}}<+\infty .
$$

Similarly, we define the weighted Sobolev space of $W^{1, p}(\Omega, \omega)$, as the space of all realvalued functions $u \in L^{p}\left(\Omega, \omega_{0}\right)$ such that the derivatives in the sense of distributions satisfy $\frac{\partial u}{\partial x_{i}} \in L^{p}\left(\Omega, \omega_{i}\right)$ for all $i=1, \ldots, N$. Equipped with the norm

$$
\|u\|_{1, p, \omega}=\left(\int_{\Omega}|u(x)|^{p} \omega_{0}(x) d x+\sum_{i=1}^{N} \int_{\Omega}\left|\frac{\partial u}{\partial x_{i}}\right|^{p} \omega_{i}(x) d x\right)^{\frac{1}{p}},
$$

$X=W^{1, p}(\Omega, \omega)$ is a Banach space. As we are concerned with a Dirichlet problem, we work in the space $X=W_{0}^{1, p}(\Omega, \omega)$ defined as the closure of $C_{0}^{\infty}(\Omega)$ with respect to the norm $\|\cdot\|_{1, p, \omega}$. Note that $C_{0}^{\infty}(\Omega)$ is dense in $W_{0}^{1, p}(\Omega, \omega)$ and $\left(W_{0}^{1, p}(\Omega, \omega),\|\cdot\|_{1, p, \omega}\right)$ is a reflexive Banach space. Note that the expression

$$
\|u\|_{X}=\left(\sum_{i=1}^{N} \int_{\Omega}\left|\frac{\partial u}{\partial x_{i}}\right|^{p} \omega_{i}(x) d x\right)^{\frac{1}{p}}
$$

is a norm defined on $X$ and is equivalent to the norm (3). Moreover $\left(X,\|\cdot\|_{X}\right)$ is a reflexive Banach space, and there exist a weight function $\sigma$ on $\Omega$ and a parameter $1<q<\infty$ such that the Hardy inequality

$$
\left(\int_{\Omega}|u|^{q} \sigma(x) d x\right)^{\frac{1}{q}} \leq C\left(\sum_{i=1}^{N} \int_{\Omega}\left|\frac{\partial u}{\partial x_{i}}\right|^{p} \omega_{i}(x) d x\right)^{\frac{1}{p}}
$$

holds for every $u \in X$ with a constant $C>0$ independent of $u$. Moreover, the imbedding $X \hookrightarrow L^{q}(\Omega, \sigma)$ is compact.

We recall that the dual of the weighted Sobolev spaces $W_{0}^{1, p}(\Omega, \omega)$ is equivalent to $W^{-1, p^{\prime}}\left(\Omega, \omega^{*}\right)$, where $\omega^{*}=\left\{\omega_{i}^{*}=\omega_{i}^{1-p^{\prime}} ; i=1 \ldots, N\right\}$ and $p^{\prime}=\frac{p}{p-1}$ is the conjugate of $p$. For more details we refer the reader to [13].

\section{Assumptions on the data and definition of a renormalized solution}

Throughout the paper, we assume that the following assumptions hold true: The functional $-\operatorname{div}(a(x, u, \nabla u))$ is a Leray-Lions operator defined on $W_{0}^{1, p}(\Omega, \omega)$ into $W^{-1, p^{\prime}}\left(\Omega, \omega^{*}\right)$ and where

$$
a: \Omega \times \mathbb{R} \times \mathbb{R}^{N} \longrightarrow \mathbb{R}^{N} \text { is a Carathéodory function, }
$$


which is monotone with respect to $\xi$ :

$$
\left[a(x, s, \xi)-a\left(x, s, \xi^{\prime}\right)\right]\left[\xi-\xi^{\prime}\right] \geq 0 ;
$$

for any $\xi, \xi^{\prime} \in \mathbb{R}^{N}$, for any $s \in \mathbb{R}$ and for almost every $x \in \Omega$, and which satisfies the following weak coercivity condition: there exists a positive function $b \in C^{0}(\mathbb{R})$ such that for almost every $x \in \Omega$, for every $s \in \mathbb{R}$ and $\xi \in \mathbb{R}^{N}$,

$$
a(x, s, \xi) \cdot \xi \geq b(s)^{p-1} \sum_{i=1}^{N} \omega_{i}(x)\left|\xi_{i}\right|^{p} \quad \text { and } \quad \int_{-\infty}^{+\infty} b(s) d s<+\infty .
$$

Moreover, $a$ satisfies a growth condition of this type: for any $i=1, \ldots, N$

$$
\begin{aligned}
& \left|a_{i}(x, s, \xi)\right| \\
& \quad \leq \omega_{i}(x)^{\frac{1}{p}}\left[L(x)+\sigma(x)^{\frac{1}{p^{\prime}}}\left(\int_{0}^{s} b(r) d r\right)^{\frac{q}{p^{\prime}}}+b(s)^{p-1} \sum_{j=1}^{N} \omega_{j}^{\frac{1}{p^{\prime}}}(x)\left|\xi_{j}\right|^{p-1}\right]
\end{aligned}
$$

and the normalization condition $a(x, s, 0)=0$ for almost every $x \in \Omega$, for every $s$ and $\xi$, and where $L(x)$ is a positive function in $L^{p^{\prime}}(\Omega)$ and $\sigma(x)$ is defined in (4).

We will study the problem in the general framework, i.e.,

$$
f \quad \text { is an element of } L^{1}(\Omega) .
$$

Remark 2.1. As already mentioned in the introduction Problem (1), (2) does not admit a weak solution under assumptions (5)-(9). Indeed, as the growth of $a(x, u, \nabla u)$ is not controlled with respect to $u$, the field $a(x, u, \nabla u)$ is not, in general, defined as a distribution.

The following notations will be used throughout the paper: for any $K \geq 0$, the truncation at height $K$ is defined by $T_{K}(r)=\max (-K, \min (r, K))$. Moreover, for $n \geq 1$ fixed,

$$
\theta_{n}(r)=T_{1}\left(r-T_{n}(r)\right)=\left\{\begin{array}{lll}
0 & \text { if } & |r| \leq n, \\
r-n s g(r) & \text { if } & n \leq|r| \leq n+1, \\
s g(r) & \text { if } & |r| \geq n+1
\end{array}\right.
$$

and $S_{n}(r)=1-\left|\theta_{n}(r)\right|, \forall r \in \mathbb{R}$.

We define a renormalized solution for Problem (1), (2) as follows.

Definition 2.2. A measurable function $u$ defined on $\Omega$ with values in $\mathbb{R} \cup\{+\infty\} \cup$ $\{-\infty\}$ is a renormalized solution of Problem (1), (2) if

$$
\begin{gathered}
T_{K}(u) \in W_{0}^{1, p}(\Omega, \omega) \quad \forall K \geq 0, \\
\int_{\{n \leq|u(x)| \leq n+1\}} a(x, u, \nabla u) \nabla u d x \longrightarrow \int_{\{u=+\infty\}} f(x) d x-\int_{\{u=-\infty\}} f(x) d x,
\end{gathered}
$$


as $n \rightarrow+\infty$, and if, for every function $S$ in $W^{1, \infty}(\mathbb{R}) \operatorname{such}$ that $\operatorname{supp}(S)$ is compact, $u$ satisfies

$$
\int_{\Omega} a(x, u, \nabla u) \nabla(S(u) \varphi) d x=\int_{\Omega} f S(u) \varphi d x, \quad \forall \varphi \in W_{0}^{1, p}(\Omega, \omega) \cap L^{\infty}(\Omega) .
$$

The following remarks are concerned with a few comments on Definition 2.2.

Remark 2.3. Notice that, thanks to our regularity assumptions (10) and the choice of $S$, all terms in (12) are well defined.

The following identifications are made in (12).

- $a(x, u, \nabla u) \nabla(S(u) \varphi)$ identifies with $a\left(x, T_{K}(u), \nabla T_{K}(u)\right) \nabla\left(S\left(T_{K}(u)\right) \varphi\right)$ a.e. in $\Omega$, where $K>0$ and $\operatorname{supp}(S) \subset[-K, K]$. As a consequence of (8), (10) and of $S \in W^{1, \infty}(\mathbb{R}), \varphi \in W_{0}^{1, p}(\Omega, \omega) \cap L^{\infty}(\Omega)$, it follows that

$$
a\left(x, T_{K}(u), \nabla T_{K}(u)\right) \nabla\left(S\left(T_{K}(u)\right) \varphi\right) \in L^{1}(\Omega) .
$$

Indeed,

$$
\nabla(S(u) \varphi)=\nabla\left(S\left(T_{K}(u)\right) \varphi\right) \in \prod_{i=1}^{N} L^{p}\left(\Omega, \omega_{i}\right)
$$

and, by Holder inequality, we have, for $i=1, \ldots, N$,

$$
\begin{aligned}
\mid a_{i}\left(x, T_{K}(u),\right. & \left.\nabla T_{K}(u)\right) \mid \\
& \leq \omega_{i}(x)^{\frac{1}{p}}\left[L(x)+\sigma(x)^{\frac{1}{p^{\prime}}} C_{1}^{\frac{q}{p^{\prime}}}+C_{2}^{p-1} \sum_{j=1}^{N} \omega_{j}^{\frac{1}{p^{\prime}}}(x)\left|\frac{\partial T_{K}(u)}{\partial x_{j}}\right|^{p-1}\right]
\end{aligned}
$$

where $C_{1}=\int_{-\infty}^{+\infty} b(s) d s$ and $C_{2}=\max _{|s| \leq K}|b(s)|$.

- $f S(u) \varphi \in L^{1}(\Omega)$, because $f \in L^{1}(\Omega)$ and $S(u) \varphi \in L^{\infty}(\Omega)$.

\section{Existence result}

This section is devoted to establish the existence theorem.

Theorem 3.1. Under the assumptions (5)-(9) there exists at least a renormalized solution u of Problem (1), (2).

Proof. The proof is divided into 6 steps. In step 1, we introduce an approximate problem. Step 2 is devoted to establish a few a priori estimates on the approximate solutions $u^{\varepsilon}$ and on the limit solution $u$. In particular, we prove that $u$ satisfies (10). In step 3, we prove the monotonicity estimate. In step 4, we identify the weak limit $X_{K}$ of $a^{\varepsilon}\left(x, T_{K}\left(u^{\varepsilon}\right), \nabla T_{K}\left(u^{\varepsilon}\right)\right)$ and we prove the weak $L^{1}$ convergence of the 
energy $a^{\varepsilon}\left(x, T_{K}\left(u^{\varepsilon}\right), \nabla T_{K}\left(u^{\varepsilon}\right)\right) \nabla T_{K}\left(u^{\varepsilon}\right)$ as $\varepsilon$ tends to zero. Step 5 is devoted to prove that $u$ satisfies (11). Finally, in step 6, we prove that $u$ satisfies (12) of Definition 2.2.

- Step 1. We proceed by approximation: for $\varepsilon>0$, define the regularized functions

$$
\begin{gathered}
b^{\varepsilon}: \mathbb{R} \longrightarrow \mathbb{R}, r \longmapsto b^{\varepsilon}(r)=b\left(T_{\frac{1}{\varepsilon}}(r)\right), \\
a^{\varepsilon}(x, s, \xi)=a\left(x, T_{\frac{1}{\varepsilon}}(s), \xi\right) \quad \text { a.e. in } \Omega, \quad \forall s \in \mathbb{R}, \quad \forall \xi \in \mathbb{R}^{N}, \\
f^{\varepsilon} \in L^{p^{\prime}}(\Omega), \quad\left\|f^{\varepsilon}\right\|_{L^{1}(\Omega)} \leq\|f\|_{L^{1}(\Omega)}, \\
f^{\varepsilon} \longrightarrow f \quad \text { strongly in } L^{1}(\Omega) \text { as } \varepsilon \text { tends to } 0,
\end{gathered}
$$

Let us now consider the following regularized problem:

$$
\begin{aligned}
-\operatorname{div}\left(a^{\varepsilon}\left(x, u^{\varepsilon}, \nabla u^{\varepsilon}\right)\right) & =f^{\varepsilon} & & \text { in } \Omega, \\
u^{\varepsilon} & =0 & & \text { on } \partial \Omega .
\end{aligned}
$$

In view of (7), (8), (13), and (14), $b^{\varepsilon}$ and $a^{\varepsilon}$ satisfy

$$
\begin{aligned}
& 0<\alpha_{\varepsilon} \equiv \min _{\left\{|r| \leq \frac{1}{\varepsilon}\right\}}(b(r)) \leq b^{\varepsilon}(s) \leq \max _{\left\{|r| \leq \frac{1}{\varepsilon}\right\}}(b(r)) \equiv C_{\varepsilon} \quad \forall s \in \mathbb{R} . \\
& a^{\varepsilon}(x, s, \xi) \cdot \xi \geq b^{\varepsilon}(s)^{p-1} \sum_{i=1}^{N} \omega_{i}(x)\left|\xi_{i}\right|^{p} \geq \alpha_{\varepsilon}^{p-1} \sum_{i=1}^{N} \omega_{i}(x)\left|\xi_{i}\right|^{p},
\end{aligned}
$$

and, for $i=1, \ldots, N$,

$$
\left|a_{i}^{\varepsilon}(x, s, \xi)\right| \leq \omega_{i}(x)^{\frac{1}{p}}\left[L(x)+C_{\varepsilon}^{\frac{q}{p^{\prime}}} \sigma(x)^{\frac{1}{p^{\prime}}}|s|^{\frac{q}{p^{\prime}}}+C_{\varepsilon}^{p-1} \sum_{j=1}^{N} \omega_{j}^{\frac{1}{p^{\prime}}}(x)\left|\xi_{j}\right|^{p-1}\right]
$$

a.e. $x \in \Omega, \forall s \in \mathbb{R}, \xi \in \mathbb{R}^{N}$.

As a consequence, proving the existence of a weak solution $u^{\varepsilon} \in W_{0}^{1, p}(\Omega, \omega)$ of (16) and (17) is an easy task (see, e.g., Theorem 2.1 and Remark 2.1 in chapter 2 of [14] and see also [1]).

- Step 2. A priori estimates and pointwise convergence of $u^{\varepsilon}$.

Using $T_{K}\left(u^{\varepsilon}\right)$ as a test function in (16) leads to

$$
\int_{\Omega} a^{\varepsilon}\left(x, u^{\varepsilon}, \nabla u^{\varepsilon}\right) \nabla T_{K}\left(u^{\varepsilon}\right) d x=\int_{\Omega} f^{\varepsilon} T_{K}\left(u^{\varepsilon}\right) d x \leq K\|f\|_{L^{1}(\Omega)} .
$$

Since $a^{\varepsilon}$ satisfies (18), we deduce from (19) that

$$
\int_{\Omega} b^{\varepsilon}\left(u^{\varepsilon}\right)^{p-1} \sum_{i=1}^{N}\left|\frac{\partial T_{K}\left(u^{\varepsilon}\right)}{\partial x_{i}}\right|^{p} \omega_{i}(x) d x \leq K\|f\|_{L^{1}(\Omega)} .
$$


and

$$
\alpha_{\frac{1}{K}}^{p-1} \int_{\Omega} \sum_{i=1}^{N}\left|\frac{\partial T_{K}\left(u^{\varepsilon}\right)}{\partial x_{i}}\right|^{p} \omega_{i}(x) d x \leq K\|f\|_{L^{1}(\Omega)},
$$

where $\alpha_{\frac{1}{K}} \equiv \min _{\{|s| \leq K\}}(b(s))$. From (20) and (4), we deduce with a classical argument (see, e.g., [1]) that, for a subsequence still indexed by $\varepsilon$,

$$
u^{\varepsilon} \longrightarrow u \text { a.e. in } \Omega \text {, }
$$

$$
T_{K}\left(u^{\varepsilon}\right) \longrightarrow T_{K}(u) \text { weakly in } W_{0}^{1, p}(\Omega, \omega) \text { and strongly in } L^{q}(\Omega, \sigma) \text {, }
$$

as $\varepsilon$ tends to 0 , where $u$ is a measurable function defined on $\Omega$ with values in $\mathbb{R} \cup$ $\{-\infty\} \cup\{+\infty\}$.

Taking now $Z^{\varepsilon}=\int_{0}^{T_{K}\left(u^{\varepsilon}\right)} b^{\varepsilon}(s) d s$ as a test function in (16) gives

$$
\int_{\Omega} a^{\varepsilon}\left(x, u^{\varepsilon}, \nabla u^{\varepsilon}\right) \nabla Z^{\varepsilon} d x=\int_{\Omega} f^{\varepsilon} Z^{\varepsilon} d x .
$$

Since $a^{\varepsilon}$ satisfies (18), (23) leads to

$$
\int_{\Omega} \sum_{i=1}^{N}\left|\frac{\partial Z^{\varepsilon}}{\partial x_{i}}\right|^{p} \omega_{i}(x) d x \leq C_{K}\|f\|_{L^{1}(\Omega)} .
$$

where $\left|Z^{\varepsilon}\right| \leq C_{K}=2 K \max _{|s| \leq K} b(s)$ is a constant independent of $\varepsilon$.

Now, for fixed $K>0$, assumption (8) gives, for $i=1, \ldots, N$,

$$
\begin{aligned}
& \left|a_{i}^{\varepsilon}\left(x, T_{K}\left(u^{\varepsilon}\right), \nabla T_{K}\left(u^{\varepsilon}\right)\right)\right| \\
& \quad \leq \omega_{i}(x)^{\frac{1}{p}}\left[L(x)+\sigma(x)^{\frac{1}{p^{\prime}}} C_{K} \frac{q}{p^{\prime}}+\sum_{j=1}^{N} \omega_{j}^{\frac{1}{p^{\prime}}}(x)\left|\frac{\partial Z^{\varepsilon}}{\partial x_{j}}\right|^{p-1}\right]
\end{aligned}
$$

In view of (24) and (25), we deduce that

$$
a^{\varepsilon}\left(x, T_{K}\left(u^{\varepsilon}\right), \nabla T_{K}\left(u^{\varepsilon}\right)\right) \text { is bounded in } \prod_{i=1}^{N} L^{p^{\prime}}\left(\Omega, w_{i}^{1-p^{\prime}}\right),
$$

then there exists a function $X_{K} \in \prod_{i=1}^{N} L^{p^{\prime}}\left(\Omega, w_{i}^{1-p^{\prime}}\right)$ such that

$$
a^{\varepsilon}\left(x, T_{K}\left(u^{\varepsilon}\right), \nabla T_{K}\left(u^{\varepsilon}\right)\right) \longrightarrow X_{K} \quad \text { weakly in } \quad \prod_{i=1}^{N} L^{p^{\prime}}\left(\Omega, w_{i}^{1-p^{\prime}}\right) \quad \text { as } \varepsilon \rightarrow 0 .
$$

Let us now take $T_{K}\left(v^{\varepsilon}\right)$ as a test function in (16), where $v^{\varepsilon}=\int_{0}^{u^{\varepsilon}} b^{\varepsilon}(s) d s$. We obtain

$$
\int_{\Omega} a^{\varepsilon}\left(x, u^{\varepsilon}, \nabla u^{\varepsilon}\right) \nabla T_{K}\left(v^{\varepsilon}\right) d x=\int_{\Omega} f^{\varepsilon} T_{K}\left(v^{\varepsilon}\right) d x \leq K\|f\|_{L^{1}(\Omega)} .
$$


Then (18) yields

$$
\int_{\Omega} \sum_{i=1}^{N}\left|\frac{\partial T_{K}\left(v^{\varepsilon}\right)}{\partial x_{i}}\right|^{p} \omega_{i}(x) d x \leq K\|f\|_{L^{1}(\Omega)} .
$$

We deduce with a classical argument that, for a subsequence still indexed by $\varepsilon$,

$$
\begin{array}{cl}
v^{\varepsilon} \longrightarrow v & \text { a.e. in } \Omega, \\
T_{K}\left(v^{\varepsilon}\right) \longrightarrow T_{K}(v) & \text { weakly in } W_{0}^{1, p}(\Omega, \omega),
\end{array}
$$

as $\varepsilon$ tends to 0 , where $v$ is a measurable function defined on $\Omega$ which is finite a.e. in $\Omega$.

Using the admissible test function $\theta_{n}\left(v^{\varepsilon}\right)$ in $(16)$ leads to

$$
\int_{\Omega} a^{\varepsilon}\left(x, u^{\varepsilon}, \nabla u^{\varepsilon}\right) \nabla \theta_{n}\left(v^{\varepsilon}\right) d x=\int_{\Omega} f^{\varepsilon} \theta_{n}\left(v^{\varepsilon}\right) d x
$$

As a consequence of the above convergence results, we are in a position to pass to the limit as $\varepsilon$ tends to 0 in (30). Indeed, the pointwise convergence of $\theta_{n}\left(v^{\varepsilon}\right)$ to $\theta_{n}(v)$ as $\varepsilon$ tends to zero and $\left|\theta_{n}\left(v^{\varepsilon}\right)\right| \leq 1$ a.e. in $\Omega$ (independently of $\varepsilon$ and $n$ ) leads to

$$
\lim _{\varepsilon \rightarrow 0} \int_{\Omega} a^{\varepsilon}\left(x, u^{\varepsilon}, \nabla u^{\varepsilon}\right) \nabla \theta_{n}\left(v^{\varepsilon}\right) d x=\int_{\Omega} f \theta_{n}(v) d x .
$$

The pointwise convergence of $\theta_{n}(v)$ to zero as $n$ tends to $+\infty$, the bounded character of $\theta_{n}\left(\left|\theta_{n}\left(v^{\varepsilon}\right)\right| \leq 1\right.$ a.e. in $\Omega$, independently of $\varepsilon$ and $\left.n\right)$ and $f \in L^{1}(\Omega)$, Lebesgue's convergence theorem shows that $\int_{\Omega} f \theta_{n}(v) d x \rightarrow 0$, as $n$ tends to $+\infty$. Passing to the limit in (31) we obtain

$$
\lim _{n \rightarrow+\infty} \lim _{\varepsilon \rightarrow 0} \int_{\left\{n \leq\left|v^{\varepsilon}\right| \leq n+1\right\}} a^{\varepsilon}\left(x, u^{\varepsilon}, \nabla u^{\varepsilon}\right) \nabla v^{\varepsilon} d x=0 .
$$

- Step 3. In this step we prove the following monotonicity estimate:

LEMma 3.2. The subsequence of $u^{\varepsilon}$ defined in step 1 satisfies for any $K \geq 0$ :

$$
\begin{aligned}
\lim _{\varepsilon \rightarrow 0} \int_{\Omega}\left[a^{\varepsilon}\left(x, T_{K}\left(u^{\varepsilon}\right), \nabla T_{K}\left(u^{\varepsilon}\right)\right)-a^{\varepsilon}\left(x, T_{K}\left(u^{\varepsilon}\right), \nabla T_{K}(u)\right)\right] & \\
& \times\left[\nabla T_{K}\left(u^{\varepsilon}\right)-\nabla T_{K}(u)\right] d x=0
\end{aligned}
$$

Proof of Lemma 3.2. Let $K \geq 0$ be fixed. The left hand side of equality (33) is split into

$$
\begin{aligned}
\int_{\Omega}\left[a^{\varepsilon}\left(x, T_{K}\left(u^{\varepsilon}\right), \nabla T_{K}\left(u^{\varepsilon}\right)\right)-\right. & \left.a^{\varepsilon}\left(x, T_{K}\left(u^{\varepsilon}\right), \nabla T_{K}(u)\right)\right] \\
& \times\left[\nabla T_{K}\left(u^{\varepsilon}\right)-\nabla T_{K}(u)\right] d x=A_{1}^{\varepsilon}+A_{2}^{\varepsilon}+A_{3}^{\varepsilon},
\end{aligned}
$$


where

$$
\begin{aligned}
& A_{1}^{\varepsilon}=\int_{\Omega} a^{\varepsilon}\left(x, T_{K}\left(u^{\varepsilon}\right), \nabla T_{K}\left(u^{\varepsilon}\right)\right) \nabla T_{K}\left(u^{\varepsilon}\right) d x, \\
& A_{2}^{\varepsilon}=-\int_{\Omega} a^{\varepsilon}\left(x, T_{K}\left(u^{\varepsilon}\right), \nabla T_{K}\left(u^{\varepsilon}\right)\right) \nabla T_{K}(u) d x,
\end{aligned}
$$

and

$$
A_{3}^{\varepsilon}=-\int_{\Omega} a^{\varepsilon}\left(x, T_{K}\left(u^{\varepsilon}\right), \nabla T_{K}(u)\right)\left[\nabla T_{K}\left(u^{\varepsilon}\right)-\nabla T_{K}(u)\right] d x .
$$

In the sequel we pass to the limit in (34) when $\varepsilon$ tends to 0 .

- Limit of $A_{1}^{\varepsilon}$. Using the admissible test function $S_{n}\left(v^{\varepsilon}\right) T_{K}(u)$ in (16) leads to

$$
\begin{array}{r}
\int_{\Omega} S_{n}\left(v^{\varepsilon}\right) a^{\varepsilon}\left(x, u^{\varepsilon}, \nabla u^{\varepsilon}\right) \nabla T_{K}(u) d x+\int_{\Omega} a^{\varepsilon}\left(x, u^{\varepsilon}, \nabla u^{\varepsilon}\right) \nabla S_{n}\left(v^{\varepsilon}\right) \cdot T_{K}(u) d x \\
=\int_{\Omega} f^{\varepsilon} S_{n}\left(v^{\varepsilon}\right) T_{K}(u) d x
\end{array}
$$

where $v^{\varepsilon}=\int_{0}^{u^{\varepsilon}} b^{\varepsilon}(s) d s$.

Passing to the limit as $\varepsilon$ tends to 0 in $(35)$, since $\operatorname{supp}\left(S_{n}\right) \subset[-(n+1), n+1]$, we have for $i=1, \ldots, N$ that

$$
\begin{aligned}
& \left|a_{i}^{\varepsilon}\left(x, u^{\varepsilon}, \nabla u^{\varepsilon}\right) S_{n}\left(v^{\varepsilon}\right)\right| \\
& \quad \leq\left\|S_{n}\right\|_{L^{\infty}(\mathbb{R})} \omega_{i}(x)^{\frac{1}{p}}\left[L(x)+\sigma(x)^{\frac{1}{p^{\prime}}}(n+1)^{\frac{q}{p^{\prime}}}+\sum_{j=1}^{N} \omega_{j}^{\frac{1}{p^{\prime}}}(x)\left|\frac{\partial T_{n+1}\left(v^{\varepsilon}\right)}{\partial x_{j}}\right|^{p-1}\right]
\end{aligned}
$$

In view of (28) and (36), we deduce that, for fixed $n \geq 1$,

$$
a^{\varepsilon}\left(x, u^{\varepsilon}, \nabla u^{\varepsilon}\right) S_{n}\left(v^{\varepsilon}\right) \text { is bounded in } \prod_{i=1}^{N} L^{p^{\prime}}\left(\Omega, w_{i}^{1-p^{\prime}}\right),
$$

independently of $\varepsilon$. Then there exists a function $Y_{n} \in \prod_{i=1}^{N} L^{p^{\prime}}\left(\Omega, w_{i}^{1-p^{\prime}}\right)$ such that, for fixed $n \geq 1$,

$$
S_{n}\left(v^{\varepsilon}\right) a^{\varepsilon}\left(x, u^{\varepsilon}, \nabla u^{\varepsilon}\right) \longrightarrow Y_{n} \quad \text { weakly in } \prod_{i=1}^{N} L^{p^{\prime}}\left(\Omega, w_{i}^{1-p^{\prime}}\right) \quad \text { as } \varepsilon \rightarrow 0 \text {. }
$$

Now, for $K \leq n$ we have

$$
S_{n}\left(v^{\varepsilon}\right) a^{\varepsilon}\left(x, u^{\varepsilon}, \nabla u^{\varepsilon}\right) \chi_{\left\{\left|u^{\varepsilon}\right| \leq K\right\}}=S_{n}\left(v^{\varepsilon}\right) a^{\varepsilon}\left(x, T_{K}\left(u^{\varepsilon}\right), \nabla T_{K}\left(u^{\varepsilon}\right)\right)
$$


a.e. in $\Omega$, which implies that, through the use of (27), (29), and (37), and passing to the limit as $\varepsilon$ tends to 0 ,

$$
Y_{n} \chi_{\{|u|<K\}}=S_{n}(v) X_{K}
$$

a.e. in $\Omega \backslash\{|u|=K\}$. As a consequence of (37) we have for $K \leq n$ that

$$
Y_{n} \nabla T_{K}(u)=S_{n}(v) X_{K} \nabla T_{K}(u) \text { a.e. in } \Omega .
$$

We are now in a position to exploit (35), which together with (37) and (39), gives

$$
\begin{aligned}
\lim _{\varepsilon \rightarrow 0} \int_{\Omega} S_{n}\left(v^{\varepsilon}\right) a^{\varepsilon}\left(x, u^{\varepsilon}, \nabla u^{\varepsilon}\right) & \nabla T_{K}(u) d x \\
& =\int_{\Omega} Y_{n} \nabla T_{K}(u) d x=\int_{\Omega} S_{n}(v) X_{K} \nabla T_{K}(u) d x
\end{aligned}
$$

Passing to the limit as $n$ tends to $+\infty$ in (40) leads to

$$
\lim _{n \rightarrow+\infty} \lim _{\varepsilon \rightarrow 0} \int_{\Omega} S_{n}\left(v^{\varepsilon}\right) a^{\varepsilon}\left(x, u^{\varepsilon}, \nabla u^{\varepsilon}\right) \nabla T_{K}(u) d x=\int_{\Omega} X_{K} \nabla T_{K}(u) d x
$$

Now, we estimate the second term of (35):

$$
\begin{aligned}
&\left|\int_{\Omega} a^{\varepsilon}\left(x, u^{\varepsilon}, \nabla u^{\varepsilon}\right) \nabla S_{n}\left(v^{\varepsilon}\right) \cdot T_{K}(u) d x\right| \\
& \leq K \int_{\left\{n \leq\left|v^{\varepsilon}\right| \leq n+1\right\}} a^{\varepsilon}\left(x, u^{\varepsilon}, \nabla u^{\varepsilon}\right) \nabla v^{\varepsilon} d x .
\end{aligned}
$$

Then (32) implies that

$$
\lim _{n \rightarrow+\infty} \lim _{\varepsilon \rightarrow 0} \int_{\Omega} a^{\varepsilon}\left(x, u^{\varepsilon}, \nabla u^{\varepsilon}\right) \nabla S_{n}\left(v^{\varepsilon}\right) \cdot T_{K}(u) d x=0 .
$$

In view of (41) and (42), passing to the limit as $\varepsilon$ tends to 0 and $n$ tends to $+\infty$ in (35) is an easy task and leads to

$$
\int_{\Omega} X_{K} \nabla T_{K}(u) d x=\int_{\Omega} f T_{K}(u) d x
$$

We are now in a position to exploit (43). Using the test function $T_{K}\left(u^{\varepsilon}\right)$ in (16), we obtain

$$
\int_{\Omega} a^{\varepsilon}\left(x, u^{\varepsilon}, \nabla u^{\varepsilon}\right) \nabla T_{K}\left(u^{\varepsilon}\right) d x=\int_{\Omega} f^{\varepsilon} T_{K}\left(u^{\varepsilon}\right) d x
$$

Passing to the limit as $\varepsilon$ tends to 0 in (44). In view (43), we have

$$
\lim _{\varepsilon \rightarrow 0} A_{1}^{\varepsilon}=\lim _{\varepsilon \rightarrow 0} \int_{\Omega} a^{\varepsilon}\left(x, u^{\varepsilon}, \nabla u^{\varepsilon}\right) \nabla T_{K}\left(u^{\varepsilon}\right) d x=\int_{\Omega} X_{K} \nabla T_{K}(u) d x
$$


- Limit of $A_{2}^{\varepsilon}$. In view of (21), (26), we have

$$
\lim _{\varepsilon \rightarrow 0} A_{2}^{\varepsilon}=-\int_{\Omega} X_{K} \nabla T_{K}(u) d x
$$

- Limit of $A_{3}^{\varepsilon}$. Let us remark that (5), (14), and (21) imply that

$$
a^{\varepsilon}\left(x, T_{K}\left(u^{\varepsilon}\right), \nabla T_{K}(u)\right) \rightarrow a\left(x, T_{K}(u), \nabla T_{K}(u)\right) \text { a.e. in } \Omega,
$$

as $\varepsilon$ tends to 0 , and that, for $i=1, \ldots, N$,

$$
\begin{aligned}
& \left|a_{i}^{\varepsilon}\left(x, T_{K}\left(u^{\varepsilon}\right), \nabla T_{K}(u)\right)\right| \\
& \quad \leq w_{i}^{\frac{1}{p}}(x)\left[L(x)+\sigma^{\frac{1}{p^{\prime}}}(x)\left(\int_{-K}^{K} b(s) d s\right)^{\frac{q}{p^{\prime}}}+C_{K}^{p-1} \sum_{j=1}^{N} w_{j}^{\frac{1}{p^{\prime}}}\left|\frac{\partial T_{K}(u)}{\partial x_{j}}\right|^{p-1}\right]
\end{aligned}
$$

a.e. in $\Omega$, uniformly with respect to $\varepsilon$ and where $C_{K}=\max _{|s| \leq K}(b(s))$.

It follows that

$$
\begin{aligned}
& a^{\varepsilon}\left(x, T_{K}\left(u^{\varepsilon}\right), \nabla T_{K}(u)\right) \longrightarrow a\left(x, T_{K}(u), \nabla T_{K}(u)\right) \\
& \text { strongly in } \prod_{i=1}^{N} L^{p^{\prime}}\left(\Omega, w_{i}^{1-p^{\prime}}\right),
\end{aligned}
$$

as $\varepsilon$ tends to 0 . In view of $(21)$, we conclude that

$$
\left(\nabla T_{K}\left(u^{\varepsilon}\right)-\nabla T_{K}(u)\right) \longrightarrow 0 \text { weakly in } \prod_{i=1}^{N} L^{p}\left(\Omega, w_{i}\right), \text { as } \varepsilon \text { goes to } 0 .
$$

As a consequence of (47) and (48) we have for all $K \geq 0$ that

$$
\lim _{\varepsilon \rightarrow 0} A_{3}^{\varepsilon}=0 .
$$

In view of (45), (46), and (49), we can pass to the limit as $\varepsilon$ tends to zero in (34) and obtain (33) of Lemma 3.2.

- Step 4. In this step we identify the weak limit $X_{K}$ and we prove the weak $L^{1}$ convergence of the "truncated" energy $a^{\varepsilon}\left(x, T_{K}\left(u^{\varepsilon}\right), \nabla T_{K}\left(u^{\varepsilon}\right)\right) \nabla T_{K}\left(u^{\varepsilon}\right)$ as $\varepsilon$ tends to 0 .

Lemma 3.3. For fixed $K \geq 0$, we have

$$
X_{K}=a\left(x, T_{K}(u), \nabla T_{K}(u)\right) \quad \text { a.e. in } \Omega \text {. }
$$

And, as $\varepsilon$ tends to 0 ,

$$
\begin{array}{r}
a^{\varepsilon}\left(x, T_{K}\left(u^{\varepsilon}\right), \nabla T_{K}\left(u^{\varepsilon}\right)\right) \nabla T_{K}\left(u^{\varepsilon}\right) \longrightarrow a\left(x, T_{K}(u), \nabla T_{K}(u)\right) \\
\\
\text { weakly in } L^{1}(\Omega) .
\end{array}
$$


Proof of Lemma 3.3. Let $K \geq 0$ be fixed. In view of (22) and (26) it is possible to obtain from (33) of Lemma 3.2 that

$$
\lim _{\varepsilon \rightarrow 0} \int_{\Omega} a^{\varepsilon}\left(x, T_{K}\left(u^{\varepsilon}\right), \nabla T_{K}\left(u^{\varepsilon}\right)\right) \nabla T_{K}\left(u^{\varepsilon}\right) d x=\int_{\Omega} X_{K} \nabla T_{K}(u) d x .
$$

We use the monotone character $a$ (with respect to $\xi$ ) and for all $\psi \in \prod_{i=1}^{N} L^{p}\left(\Omega, w_{i}\right)$ we have

$$
\begin{aligned}
0 \leq & \lim _{\varepsilon \rightarrow 0} \int_{\Omega}\left[a^{\varepsilon}\left(x, T_{K}\left(u^{\varepsilon}\right), \nabla T_{K}\left(u^{\varepsilon}\right)\right)-a^{\varepsilon}\left(x, T_{K}\left(u^{\varepsilon}\right), \psi\right)\right]\left[\nabla T_{K}\left(u^{\varepsilon}\right)-\psi\right] d x \\
= & \lim _{\varepsilon \rightarrow 0} \int_{\Omega} a^{\varepsilon}\left(x, T_{K}\left(u^{\varepsilon}\right), \nabla T_{K}\left(u^{\varepsilon}\right)\right)\left[\nabla T_{K}\left(u^{\varepsilon}\right)-\psi\right] d x \\
& -\lim _{\varepsilon \rightarrow 0} \int_{\Omega} a^{\varepsilon}\left(x, T_{K}\left(u^{\varepsilon}\right), \psi\right)\left[\nabla T_{K}\left(u^{\varepsilon}\right)-\psi\right] d x \\
= & \int_{\Omega} X_{K}\left[\nabla T_{K}(u)-\psi\right] d x-\int_{\Omega} a\left(x, T_{K}(u), \psi\right)\left[\nabla T_{K}(u)-\psi\right] d x \\
= & \int_{\Omega}\left[X_{K}-a\left(x, T_{K}(u), \psi\right)\right]\left[\nabla T_{K}(u)-\psi\right] d x
\end{aligned}
$$

The usual Minty's argument applies in view of (52). It follows that (50) of Lemma 3.3 holds true.

In order to prove (51), we use the monotone character of $a$ (with respect to $\xi$ ) and (33) to have for any $K \geq 0$ that

$$
\left(a^{\varepsilon}\left(x, T_{K}\left(u^{\varepsilon}\right), \nabla T_{K}\left(u^{\varepsilon}\right)\right)-a^{\varepsilon}\left(x, T_{K}\left(u^{\varepsilon}\right), \nabla T_{K}(u)\right)\right)\left[\nabla T_{K}\left(u^{\varepsilon}\right)-\nabla T_{K}(u)\right]
$$

converges to zero, strongly in $L^{1}(\Omega)$ as $\varepsilon$ tends to 0 . Moreover (22), (25), (47), and (50) imply that

$$
a^{\varepsilon}\left(x, T_{K}\left(u^{\varepsilon}\right), \nabla T_{K}\left(u^{\varepsilon}\right)\right) \nabla T_{K}(u) \longrightarrow a\left(x, T_{K}(u), \nabla T_{K}(u)\right) \nabla T_{K}(u)
$$

weakly in $L^{1}(\Omega)$ as tends to 0 ,

$$
a^{\varepsilon}\left(x, T_{K}\left(u^{\varepsilon}\right), \nabla T_{K}(u)\right) \nabla T_{K}\left(u^{\varepsilon}\right) \longrightarrow a\left(x, T_{K}(u), \nabla T_{K}(u)\right) \nabla T_{K}(u)
$$

weakly in $L^{1}(\Omega)$ as tends to 0 , and

$$
a^{\varepsilon}\left(x, T_{K}\left(u^{\varepsilon}\right), \nabla T_{K}(u)\right) \nabla T_{K}(u) \longrightarrow a\left(x, T_{K}(u), \nabla T_{K}(u)\right) \nabla T_{K}(u)
$$

strongly in $L^{1}(\Omega)$ as tends to 0 .

Using the above convergence results (54), (55), and (56) in (53) we get, for any $K \geq 0$,

$$
a^{\varepsilon}\left(x, T_{K}\left(u^{\varepsilon}\right), \nabla T_{K}\left(u^{\varepsilon}\right)\right) \nabla T_{K}\left(u^{\varepsilon}\right) \longrightarrow a\left(x, T_{K}(u), \nabla T_{K}(u)\right) \nabla T_{K}(u)
$$

weakly in $L^{1}(\Omega)$ as tends to 0 . 
- Step 5. In this step we prove that $u$ satisfies (11).

Using $\left(T_{n+1}\left(u^{\varepsilon}\right)-T_{n}\left(u^{\varepsilon}\right)\right) S_{p}\left(v^{\varepsilon}\right)$ as a test function in (16) leads to

$$
\begin{gathered}
\int_{\Omega} S_{p}\left(v^{\varepsilon}\right) a^{\varepsilon}\left(x, u^{\varepsilon}, \nabla u^{\varepsilon}\right) \nabla\left(T_{n+1}\left(u^{\varepsilon}\right)-T_{n}\left(u^{\varepsilon}\right)\right) d x \\
+\int_{\Omega}\left(T_{n+1}\left(u^{\varepsilon}\right)-T_{n}\left(u^{\varepsilon}\right)\right) a^{\varepsilon}\left(x, u^{\varepsilon}, \nabla u^{\varepsilon}\right) \nabla S_{p}\left(v^{\varepsilon}\right) d x \\
=\int_{\Omega} f^{\varepsilon} S_{p}\left(v^{\varepsilon}\right)\left(T_{n+1}\left(u^{\varepsilon}\right)-T_{n}\left(u^{\varepsilon}\right)\right) d x .
\end{gathered}
$$

Remark that for any fixed $n \geq 0$ and $p \geq 0$ one has

$$
\begin{gathered}
\int_{\left\{n \leq\left|u^{\varepsilon}(x)\right| \leq n+1\right\}} S_{p}\left(v^{\varepsilon}\right) a^{\varepsilon}\left(x, u^{\varepsilon}, \nabla u^{\varepsilon}\right) \nabla u^{\varepsilon} d x \\
=\int_{\Omega} S_{p}\left(v^{\varepsilon}\right) a^{\varepsilon}\left(x, T_{n+1}\left(u^{\varepsilon}\right), \nabla T_{n+1}\left(u^{\varepsilon}\right)\right) \nabla T_{n+1}\left(u^{\varepsilon}\right) d x \\
\quad-\int_{\Omega} S_{p}\left(v^{\varepsilon}\right) a^{\varepsilon}\left(x, T_{n}\left(u^{\varepsilon}\right), \nabla T_{n}\left(u^{\varepsilon}\right)\right) \nabla T_{n}\left(u^{\varepsilon}\right) d x .
\end{gathered}
$$

According to (51), one can pass to the limit as $\varepsilon$ tends to zero for fixed $n$ and $p$ to obtain

$$
\begin{aligned}
& \lim _{\varepsilon \rightarrow 0} \int_{\left\{n \leq\left|u^{\varepsilon}(x)\right| \leq n+1\right\}} S_{p}\left(v^{\varepsilon}\right) a^{\varepsilon}\left(x, u^{\varepsilon}, \nabla u^{\varepsilon}\right) \nabla u^{\varepsilon} d x \\
&= \int_{\Omega} S_{p}(v) a\left(x, T_{n+1}(u), \nabla T_{n+1}(u)\right) \nabla T_{n+1}(u) d x \\
&-\int_{\Omega} S_{p}(v) a\left(x, T_{n}(u), \nabla T_{n}(u)\right) \nabla T_{n}(u) d x \\
&= \int_{\{n \leq|u(x)| \leq n+1\}} S_{p}(v) a(x, u, \nabla u) \nabla u d x .
\end{aligned}
$$

Taking the limit as $p$ tends to $+\infty$ and as $n$ tends to $+\infty$ in (57) we obtain

$$
\begin{aligned}
\lim _{n \rightarrow+\infty} \lim _{p \rightarrow+\infty} \lim _{\varepsilon \rightarrow 0} \int_{\Omega} S_{p}\left(v^{\varepsilon}\right) a^{\varepsilon}\left(x, u^{\varepsilon}, \nabla u^{\varepsilon}\right) & \nabla\left(T_{n+1}\left(u^{\varepsilon}\right)-T_{n}\left(u^{\varepsilon}\right)\right) d x \\
= & \lim _{n \rightarrow+\infty} \int_{\{n \leq|u(x)| \leq n+1\}} a(x, u, \nabla u) \nabla u d x
\end{aligned}
$$

Since $\operatorname{supp}\left(S_{p}^{\prime}\right) \subset[-(p+1),-p] \cup[p, p+1]$, we have

$$
\begin{aligned}
\mid \int_{\Omega}\left(T_{n+1}\left(u^{\varepsilon}\right)-T_{n}\left(u^{\varepsilon}\right)\right) a^{\varepsilon}\left(x, u^{\varepsilon}, \nabla u^{\varepsilon}\right) & \nabla S_{p}\left(v^{\varepsilon}\right) d x \mid \\
& \leq \int_{\left\{p \leq\left|v^{\varepsilon}(x)\right| \leq p+1\right\}}\left|a^{\varepsilon}\left(x, u^{\varepsilon}, \nabla u^{\varepsilon}\right) \nabla v^{\varepsilon}\right| d x
\end{aligned}
$$


In view of (21), (29), and (32), we obtain

$$
\begin{gathered}
\lim _{n \rightarrow+\infty} \lim _{p \rightarrow+\infty} \lim _{\varepsilon \rightarrow 0}\left|\int_{\Omega}\left(T_{n+1}\left(u^{\varepsilon}\right)-T_{n}\left(u^{\varepsilon}\right)\right) a^{\varepsilon}\left(x, u^{\varepsilon}, \nabla u^{\varepsilon}\right) \nabla S_{p}\left(v^{\varepsilon}\right) d x\right|=0 \\
\lim _{n \rightarrow+\infty} \lim _{p \rightarrow+\infty} \lim _{\varepsilon \rightarrow 0} \int_{\Omega} f^{\varepsilon} S_{p}\left(v^{\varepsilon}\right)\left(T_{n+1}\left(u^{\varepsilon}\right)-T_{n}\left(u^{\varepsilon}\right)\right) d x \\
=\int_{\{u=+\infty\}} f(x) d x-\int_{\{u=-\infty\}} f(x) d x .
\end{gathered}
$$

- Step 6. In this step, $u$ is shown to satisfy (12). Let $\varphi \in W_{0}^{1, p}(\Omega, \omega) \cap L^{\infty}(\Omega)$ and let $S$ be a function in $W^{1, \infty}(\mathbb{R})$ such that $S$ has a compact support. Let $K$ be a positive real number such that $\operatorname{supp} S \subset[-K, K]$ and $v^{\varepsilon}=\int_{0}^{u^{\varepsilon}} b^{\varepsilon}(s) d s$. Using $S(u) S_{n}\left(v^{\varepsilon}\right) \varphi$ as a test function in (16) leads to

$$
\begin{array}{r}
\int_{\Omega} S_{n}\left(v^{\varepsilon}\right) a^{\varepsilon}\left(x, u^{\varepsilon}, \nabla u^{\varepsilon}\right) \nabla(S(u) \varphi) d x+\int_{\Omega} S(u) \varphi a^{\varepsilon}\left(x, u^{\varepsilon}, \nabla u^{\varepsilon}\right) \nabla S_{n}\left(v^{\varepsilon}\right) d x \\
=\int_{\Omega} f^{\varepsilon} S_{n}\left(v^{\varepsilon}\right) S(u) \varphi d x .
\end{array}
$$

In the following, we pass to the limit as $\varepsilon$ tends to 0 and $n$ tends to $+\infty$ in each term of (58).

- Limit of the first term in (58).

In view of (37), (38), and (50), passing to the limit as $\varepsilon$ tends to 0 , we get

$$
\begin{aligned}
\lim _{\varepsilon \rightarrow 0} \int_{\Omega} S_{n}\left(v^{\varepsilon}\right) a^{\varepsilon}\left(x, u^{\varepsilon}, \nabla u^{\varepsilon}\right) & \nabla(S(u) \varphi) d x \\
& =\int_{\Omega} Y_{n} \nabla(S(u) \varphi) d x=\int_{\Omega} Y_{n} \chi_{\{|u| \leq K\}} \nabla(S(u) \varphi) d x \\
& =\int_{\Omega} S_{n}(v) X_{K} \nabla(S(u) \varphi) d x \\
& =\int_{\Omega} S_{n}(v) a\left(x, T_{K}(u), \nabla T_{K}(u)\right) \nabla(S(u) \varphi) d x
\end{aligned}
$$

and

$$
\begin{aligned}
\lim _{n \rightarrow+\infty} \lim _{\varepsilon \rightarrow 0} \int_{\Omega} S_{n}\left(v^{\varepsilon}\right) a^{\varepsilon}\left(x, u^{\varepsilon},\right. & \left.\nabla u^{\varepsilon}\right) \nabla(S(u) \varphi) d x \\
& =\lim _{n \rightarrow+\infty} \int_{\Omega} S_{n}(v) a\left(x, T_{K}(u), \nabla T_{K}(u)\right) \nabla(S(u) \varphi) d x \\
& =\int_{\Omega} a\left(x, T_{K}(u), \nabla T_{K}(u)\right) \nabla(S(u) \varphi) d x \\
& =\int_{\Omega} a(x, u, \nabla u) \nabla(S(u) \varphi) d x
\end{aligned}
$$


- Limit of the second term in (58). Since $\operatorname{supp}\left(S_{n}^{\prime}\right) \subset[-(n+1),-n] \cup[n+1, n]$ for any $n \geq 1$, we have, as a consequence, that

$$
\begin{aligned}
\mid \int_{\Omega} S(u) \varphi a^{\varepsilon}\left(x, u^{\varepsilon}\right. & \left., \nabla u^{\varepsilon}\right) \nabla S_{n}\left(v^{\varepsilon}\right) d x \mid \\
& \leq\|S\|_{L^{\infty}(\mathbb{R})}\|\varphi\|_{L^{\infty}(\Omega)} \int_{\left\{n \leq\left|v^{\varepsilon}\right| \leq n+1\right\}}\left|a^{\varepsilon}\left(x, u^{\varepsilon}, \nabla u^{\varepsilon}\right) \nabla v^{\varepsilon}\right| d x .
\end{aligned}
$$

Taking the limit as $\varepsilon$ tends to 0 and $n$ tends to $+\infty$ in (59) and using the estimate (32) yields

$$
\lim _{n \rightarrow+\infty} \lim _{\varepsilon \rightarrow 0}\left|\int_{\Omega} S(u) \varphi a^{\varepsilon}\left(x, u^{\varepsilon}, \nabla u^{\varepsilon}\right) \nabla S_{n}\left(v^{\varepsilon}\right) d x\right|=0
$$

- Limit of the right-hand side of (58). Due to (15) and (29), we have

$$
\lim _{n \rightarrow+\infty} \lim _{\varepsilon \rightarrow 0} \int_{\Omega} f^{\varepsilon} S_{n}\left(v^{\varepsilon}\right) S(u) \varphi d x=\int_{\Omega} f S(u) \varphi d x .
$$

Thanks to the above convergence results, we are in a position to pass to the limit as $\varepsilon$ tends to 0 in (58) and to conclude that $u$ satisfies (12). The proof of Theorem 3.1 is achieved.

Acknowledgement. The authors would like to thank the anonymous referees for their interesting remarks.

\section{References}

[1] L. Aharouch, E. Azroul, and A. Benkirane, Quasilinear degenerated equations with $L^{1}$ datum and without coercivity in perturbation terms, Electron. J. Qual. Theory Differ. Equ. 19 (2006), $1-18$.

[2] A. Alvino, L. Boccardo, V. Ferone, L. Orsina, and G. Trombetti, Existence results for nonlinear elliptic equations with degenerate coercivity, Ann. Mat. Pura Appl. (4) 182 (2003), no. 1, 53-79.

[3] A. Alvino, V. Ferone, and G. Trombetti, A priori estimates for a class of non-uniformly elliptic equations, Atti Sem. Mat. Fis. Univ. Modena 46 (1998), no. suppl., 381-391.

[4] K. Ammar, Renormalized solutions of degenerate elliptic problems, J. Differential Equations 234 (2007), no. 1, 1-25.

[5] P. Bénilan, L. Boccardo, T. Gallouët, R. Gariepy, M. Pierre, and J. L. Vázquez, An $L^{1}$-theory of existence and uniqueness of solutions of nonlinear elliptic equations, Ann. Scuola Norm. Sup. Pisa Cl. Sci. (4) 22 (1995), no. 2, 241-273.

[6] D. Blanchard and O. Guibé, Infinite valued solutions of non-uniformly elliptic problems, Anal. Appl. (Singap.) 2 (2004), no. 3, 227-246.

[7] D. Blanchard, O. Guibé, and H. Redwane, Nonlinear equations with unbounded heat conduction and integrable data, Ann. Mat. Pura Appl. (4) 187 (2008), no. 3, 405-433. 
[8] D. Blanchard, F. Murat, and H. Redwane, Existence and uniqueness of a renormalized solution for a fairly general class of nonlinear parabolic problems, J. Differential Equations 177 (2001), no. 2, 331-374.

[9] D. Blanchard and H. Redwane, Renormalized solutions for a class of nonlinear evolution problems, J. Math. Pures Appl. (9) 77 (1998), no. 2, 117-151.

[10] L. Boccardo, A. Dall'Aglio, and L. Orsina, Existence and regularity results for some elliptic equations with degenerate coercivity, Atti Sem. Mat. Fis. Univ. Modena 46 (1998), no. suppl., $51-81$.

[11] L. Boccardo, D. Giachetti, J. I. Diaz, and F. Murat, Existence and regularity of renormalized solutions for some elliptic problems involving derivatives of nonlinear terms, J. Differential Equations 106 (1993), no. 2, 215-237.

[12] R. J. DiPerna and P.-L. Lions, On the Cauchy problem for Boltzmann equations: Global existence and weak stability, Ann. of Math. (2) 130 (1989), no. 2, 321-366.

[13] P. Drábek, A. Kufner, and F. N. Nicolosi, Non linear elliptic equations, singular and degenerate cases, University of West Bohemia, 1996.

[14] J.-L. Lions, Quelques méthodes de résolution des problèmes aux limites non linéaires, Dunod et Gauthier-Villars, Paris, 1969.

[15] P.-L. Lions, Mathematical topics in fluid mechanics. Vol. 1, Oxford Lecture Series in Mathematics and its Applications, vol. 3, The Clarendon Press Oxford University Press, New York, 1996.

[16] F. Murat, Soluciones renormalizadas de EDP elipticas non lineales, Laboratoire d'Analyse Numérique, Paris VI, 1996. Cours à l'Université de Séville.

[17]__ Equations elliptiques non linéaires avec second membre $L^{1}$ ou mesure, 26ème Congrès National d'Analyse Numérique (Les Karellis, 1994). pp. A12-A24. 